\title{
Miomectomía laparoscópica asistida por robot, cirugía laparoscópica y laparotomía: Análisis de resultados
}

\author{
Robot-assisted laparoscopic myomectomy, laparoscopic \\ surgery and laparotomy: Analysis of results \\ Radamés Rivas López, ${ }^{\star}$ José Andrés Hernández Denis, ${ }^{\ddagger}$ Jorge Román Audifred Salomón, $\$$ \\ Pablo Joaquín Cervantes Mondragón, ${ }^{\circledR}$ Rosario Durón Padillall
* Clínica de la Mujer, Subdivisión Clínica de Reproducción Asistida, Centro de Cirugía Robótica, Hospital Ángeles Pedregal. Especialidad de Biología de la Reproducción Humana, UNAM. Ciudad de México, México.
¥ Servicio de Ginecología, Hospital Central Sur de Alta Especialidad PEMEX Picacho. Ciudad de México, México.
$\S$ Servicio de Ginecología, Hospital General «Dr. Manuel Gea González». Ciudad de México, México. ף Hospital Central Sur de Alta Especialidad PEMEX Picacho. Ciudad de México, México.
" Hospital Ángeles Mocel. Ciudad de México, México.

\section{RESUMEN}

Introducción: Los leiomiomas son el tumor pélvico más común en la mujer, éstos pueden afectar la fertilidad. La miomectomía es el tratamiento de elección en pacientes con leiomiomas sintomáticos y fines reproductivos. Se pueden realizar mediante distintas técnicas, como laparotomía, laparoscópica o asistida por robot. Múltiples estudios pretenden determinar si alguno de estos abordajes resulta superior a otro. Con este estudio se busca obtener información para elegir la mejor vía de abordaje en cada paciente. Objetivo: Describir y comparar tres tipos de abordaje para la realización de miomectomía: Asistida robóticamente, laparoscópica, laparotomía. Determinar si existe superioridad entre los tres diferentes abordajes para miomectomía. Material y métodos: Estudio retrospectivo, analítico y observaciones donde se revisaron 150 casos de miomectomías en sus distintos abordajes realizados en la Unidad de Medicina Reproductiva del Hospital Ángeles Pedregal en la Ciudad de México, 50 por laparotomía, 50 laparoscópicas y 50 auxiliadas por robot. Resultados: El tiempo quirúrgico para la cirugía robótica fue de $122 \mathrm{~min}$, laparoscópica $90 \mathrm{~min}$ y laparotomía $85 \mathrm{~min}$. El sangrado transoperatorio fue de $50 \mathrm{~mL}$ para cirugía robótica contra $235 \mathrm{~mL}$ para la cirugía abierta y $90 \mathrm{~mL}$ en cirugía laparoscópica. La estancia intrahospitalaria fue menor en cualquiera de ambas técnicas

\section{ABSTRACT}

Introduction: Leiomyomas are the most common pelvic tumor in women and they can affect fertility. Myomectomy is the treatment of choice in patients with symptomatic leiomyomas and reproductive purposes. They can be performed using different techniques such as laparotomy, laparoscopic, or robot-assisted. Multiple studies attempt to determine if any of these approaches is superior to another. This study intends to obtain information to choose the best approach for each patient. Objective: Describe and compare three types of approaches to performing myomectomy: Robot-assisted, laparoscopic, laparotomy. Determine if there is any superiority between the three different approaches to myomectomy. Material and methods: Retrospective, analytical, and observational study where 150 cases of myomectomies were reviewed in their different approaches, performed in the Reproductive Medicine Unit of Hospital Angeles Pedregal in Mexico City, 50 by laparotomy, 50 laparoscopic and 50 robot-assisted. Results: The surgical time for robotic surgery was $122 \mathrm{~min}$, laparoscopic $90 \mathrm{~min}$, and laparotomy $85 \mathrm{~min}$. Transoperative bleeding was $50 \mathrm{~mL}$ for robotic surgery versus $235 \mathrm{~mL}$ for open surgery and $90 \mathrm{~mL}$ for laparoscopic surgery. Hospital stay in either of the two minimal access techniques was shorter (1 day) compared to open surgery (2 days). The need for

Recibido: 18/09/2020. Aceptado: 29/09/2020.

Correspondencia: Dr. Radamés Rivas López. Camino Sta. Teresa Núm. 1055-129, Torre Central, Col. Héroes de Padierna, 10700, La Magdalena Contreras, Ciudad de México, CDMX. Tel: 5556526319.

E-mail: radamesrl@hotmail.com

Citar como: Rivas LR, Hernández DJA, Audifred SJR, Cervantes MPJ, Durón PR. Miomectomía laparoscópica asistida por robot, cirugía laparoscópica y laparotomía: Análisis de resultados. Rev Mex Cir Endoscop. 2020; 21 (2): 86-91. https://dx.doi.org/10.35366/98912 
de mínimo acceso (1 día) respecto a cirugía abierta (2 días). La necesidad de transfusión fue mayor en la cirugía abierta $(6 \%)$. En cuanto a complicaciones, no se presentaron en ninguno de los tres tipos de abordajes quirúrgicos. La conversión a laparotomía ocurrió en dos casos de cirugía laparoscópica (4\%). Conclusión: La miomectomía laparoscópica convencional y la apoyada por robot demostraron resultados muy similares. Es necesario realizar estudios prospectivos para determinar específicamente las ventajas entre una y otra técnica. La miomectomía laparoscópica asistida con robot con sólo tres brazos es una técnica segura para el tratamiento de miomatosis uterina.

Palabras clave: Miomectomía, cirugía robótica, miomas, laparoscopía. transfusion was greater in open surgery (6\%). Regarding complications, they did not occur in any of the three types of surgical approaches. Conversion to laparotomy occurred in two cases of laparoscopic surgery (4\%). Conclusion: Conventional and robot-assisted laparoscopic myomectomy showed very similar results. Prospective studies are necessary to specifically determine the advantages between one technique and the other. Robot-assisted laparoscopic myomectomy with only three arms is a safe technique for the treatment of uterine myomatosis.

Keywords: Myomectomy, robotic surgery, fibroids, laparoscopy.

\section{INTRODUCCIÓN}

Los miomas uterinos, también conocidos como fibromas o leiomiomas, son los tumores benignos más frecuentes que aparecen en la mujer en edad reproductiva. En función de su localización, número y tamaño, los síntomas que producen varían en frecuencia y gravedad. Existe evidencia científica de que los fibromas interfieren en la migración del esperma, el transporte de ovocitos y la implantación del embrión debido a la inflamación endometrial o alteraciones vasculares que producen. Alrededor del 5 al 10\% de las mujeres infértiles tiene miomas y su aparición es el único factor anormal encontrado en hasta en el $4 \%$ de las mismas. ${ }^{1}$ En la actualidad, en mujeres en edad reproductiva con deseo gestacional y presencia de miomas, la miomectomía probablemente sea el tratamiento de elección.

La tecnología continúa evolucionando y ampliando las opciones de tratamiento conservador para las mujeres que desean la preservación de la fertilidad. Existen para tal efecto: La embolización de las arterias uterinas, el ultrasonido de alta frecuencia guiado por resonancia magnética y la ablación por radiofrecuencia. Sin embargo, la miomectomía sigue siendo el estándar de oro para las mujeres con infertilidad que padecen de miomatosis uterina y desean un embarazo posteriormente. ${ }^{2}$

La relación entre miomas y fertilidad se sigue debatiendo, en especial en lo referente a los que son intramurales. En general se acepta que los miomas submucosos disminuyen la fertilidad y que los miomas de localización subserosa tienen poca o nula influencia en este aspecto. ${ }^{3}$ No obstante, de acuerdo con algunos reportes, los miomas intramurales que no afectan a la cavidad también se relacionan con resultados reproductivos desfavorables. El análisis de estudios prospectivos y retrospectivos muestra que los fibromas intramurales que no distorsionan la cavidad tienen un efecto adverso significativo sobre las tasas de nacidos vivos en mujeres sometidas a fertilización in vitro. ${ }^{4}$

En la actualidad, existe un debate muy interesante sobre si hay mayor beneficio en realizar el abordaje de miomec- tomía laparoscópica asistida mediante robot en pacientes con deseo de fertilidad respecto a la laparoscopía o a la cirugía abierta. En este país no existe ningún estudio que compare los diferentes tipos de miomectomía en mujeres mexicanas, así como con sus resultados reproductivos correspondientes.

El objetivo principal del presente estudio fue describir y comparar los tres tipos de abordaje quirúrgico para tener más argumentos sobre la decisión a tomar acerca de cuál vía es la más favorable y en qué casos, para la realización de una miomectomía: Abordaje robótico, abordaje laparoscópico o abordaje mediante laparotomía.

\section{MATERIAL Y MÉTODOS}

Estudio de cohorte, retrospectivo, analítico y observacional, en donde se analizaron 150 casos de miomectomías en sus distintos abordajes: Laparotomía, laparoscopía y laparoscopía asistida por robot, realizados de enero 2015 a diciembre 2019 en la Unidad de Medicina Reproductiva del Hospital Ángeles Pedregal, en la Ciudad de México.

Se incluyeron 50 pacientes con infertilidad y miomatosis uterina que fueron sometidas a laparotomía, del mismo modo, se incluyeron 50 casos con infertilidad y miomatosis uterina que fueron sometidas a cirugía laparoscópica convencional y otros 50 casos de similares antecedentes para miomectomía laparoscópica asistida por robot.

Se describen rangos, promedios y medianas. Se efectuó el análisis de desviación estándar para cada una de las variables que se tomaron en cuenta para el análisis de este trabajo.

\section{RESULTADOS}

Se analizaron un total de 150 casos de miomectomías: 50 casos realizados por cirugía robótica, 50 casos por laparoscopía y 50 más por laparotomía. Las características demográficas de las pacientes se describen en la Tabla 1. 
La media de edad fue muy similar en los tres grupos, entre 34 y 35 años, tomando en cuenta que todas las pacientes tenían deseo de embarazo a corto plazo.

También en los tres grupos el índice de masa corporal fue muy semejante: Entre 25 y 26. Los embarazos previos fueron bajos en los tres grupos, las pacientes con miomectomía previa estuvieron entre el 1 y $2 \%$. Todas las pacientes cursaban con infertilidad, ya que la institución de referencia es un centro de fertilidad. La sintomatología preoperatoria, como el sangrado, fue más frecuente en el grupo de pacientes de laparoscopía $50 \%$ y el dolor pélvico fue más frecuente en el grupo de cirugía robótica $38 \%$. Sin embargo, no fueron tan variables entre los tres grupos.

En cuanto a la clasificación de miomas de acuerdo con la Federación Internacional de Ginecología y Obstetricia, FIGO, se encontraron: Tumores submucosos en dos (1\%) pacientes, intramural exclusivo en un total de $34(22 \%)$ pacientes e híbrido en un total de 114 (76\%) pacientes. En cuanto al número máximo y mínimo de miomas extraídos, se observa un promedio de cinco miomas, con un rango de 1 hasta 30 miomas. La mayor cantidad de miomas fue extraída por la técnica de laparotomía. El mínimo de miomas para ambos procedimientos fue de 1 , frente a laparotomía con 30, laparoscopía convencional 10 y cirugía robótica 20. El tamaño o diámetro medio de los miomas fue de $9 \mathrm{~cm}$. Por el peso de los miomas, en total se registró un promedio de 480 gramos, con un rango de $10 \mathrm{~g}$ hasta $5,540 \mathrm{~g}$.

Los resultados transoperatorios entre las tres técnicas se resumen en la Tabla 2. Se observa que en tiempo quirúrgico la cirugía robótica consumió ligeramente más respecto a las otras dos técnicas: 37 minutos más que la cirugía abierta y 32 minutos más que la laparoscópica. En contraste, el sangrado transoperatorio fue muy superior, con $50 \mathrm{~mL}$ como media contra $235 \mathrm{~mL}$ para la cirugía abierta y $90 \mathrm{~mL}$ que la cirugía laparoscópica. En cuanto a estancia intrahospitalaria, los tiempos de estancia son superiores a la cirugía abierta frente a cualquiera de los abordajes de mínimo acceso. La necesidad de transfusión también fue mayor en la cirugía abierta. En cuanto a complicaciones, no se presentaron en ninguno de los tres tipos de abordajes quirúrgicos. La conversión a laparotomía sólo ocurrió en dos casos de cirugía laparoscópica.

\section{DISCUSIÓN}

El impacto de los miomas intramurales que no distorsionan la cavidad endometrial ha sido punto de controversia constante, sobre todo al elegir la estrategia terapéutica más adecuada, y como ocurrió en este estudio, los miomas intramurales son los que se encontraron con mayor frecuencia en las pacientes, sin embargo, es importante tener en cuenta la tasa de nacidos vivos y no sólo la tasa de embarazo posquirúrgica, pues los miomas se pueden relacionar con un desenlace obstétrico desfavorable. Un metaanálisis, realizado por Sunkara y Rikhraj años más tarde, señala que la presencia de miomas intramurales que no distorsionan la cavidad está asociada con un resultado adverso en mujeres sometidas a tratamiento de fertilización in vitro (FIV), se menciona que la tasa de recién nacido vivo en pacientes con miomas es un $21 \%$ menor que en mujeres sin miomas. ${ }^{3,4}$

Sobre aquellos miomas intramurales que no afectan a la cavidad, su tamaño sigue siendo punto de debate, por lo general, se toma como punto de corte para miomectomía aquellos miomas intramurales que no afectan a la cavidad pero que son mayores o igual a $4 \mathrm{~cm} ., 56$ Como ocurrió en el citado grupo de pacientes, todos los miomas intramurales medían al menos 4 o más centímetros. En estos casos, la vía de elección para el abordaje quirúrgico es de preferencia la cirugía de mínimo acceso.

\begin{tabular}{lccc} 
& Tabla 1: Datos demográficos y sintomatología preoperatoria. \\
& $\begin{array}{c}\text { Abdominal } \\
(n=50)\end{array}$ & $\begin{array}{c}\text { Laparoscópica } \\
(\mathrm{n}=50)\end{array}$ & $\begin{array}{c}\text { Robótica } \\
(\mathrm{n}=50)\end{array}$ \\
\cline { 2 - 3 } & $\mathrm{n}(\%)$ & $\mathrm{n}(\%)$ & $\mathrm{n}(\%)$ \\
\cline { 2 - 3 } & $35(26-44)$ & $34(28-41)$ & $35(25-43)$ \\
Edad, mediana (rango) & $26(18-33)$ & $25(19-32)$ & $26(19-33)$ \\
Cirugías previas & $6(12)$ & $5(10)$ & $4(8)$ \\
Embarazos previos & $2(4)$ & $1(2)$ & $2(4)$ \\
Miomectomía previa & $2(4)$ & $1(2)$ & $1(2)$ \\
Infertilidad & $50(100)$ & $50(100)$ & $50(100)$ \\
Sangrado anormal & $22(44)$ & $25(50)$ & $23(46)$ \\
Dolor & $18(36)$ & $17(34)$ & $19(38)$ \\
\hline
\end{tabular}


La vía quirúrgica de elección depende de dos factores fundamentales: En primer término, del mioma en sí, su localización, su número, tamaño y, en segundo lugar, de la experiencia del equipo quirúrgico. Los miomas de localización submucosa se tratan por vía histeroscópica, mientras que los miomas intramurales y subserosos se pueden tratar por vía laparoscópica, robótica, abdominal o vaginal. Siempre que sea posible, la vía de elección deberá ser mediante cirugía de mínimo acceso, ya que existe una base científica sólida en la que se demuestra que, con el abordaje laparoscópico robótico, laparoscópico convencional o vaginal, hay menor pérdida de sangre transoperatoria, menor tasa de adherencias, menor morbilidad posoperatoria y menos días de hospitalización, que con la miomectomía abierta. ${ }^{7}$ Es conveniente señalar que en un estudio reciente se reportó un retorno de las pacientes más rápido a sus actividades laborales y/o cotidianas después de una miomectomía por cirugía de mínimo acceso. ${ }^{8}$

En este mismo sentido, con un equipo quirúrgico que opera en forma frecuente, la miomectomía robótica es superior frente a la miomectomía laparoscópica convencional, incluso en el rubro referente al costo/beneficio. ${ }^{9} \mathrm{Y}$ es que si se cuenta con un equipo formado por anestesiólogos, médico cirujanos, enfermería y paramédicos que de forma regular realizan este tipo de abordaje robótico, se logra eficiencia, economía, menores tiempos quirúrgicos y los resultados son adecuados. Aun así, como lo menciona Wu, se necesitan más estudios para determinar qué pacientes son los que se beneficiarían enormemente por medio de un abordaje robótico, tanto en términos de resultados en la paciente como de rentabilidad. ${ }^{10}$

Takmaz y col. compararon la severidad de los síntomas y los resultados de la calidad de la salud de las mujeres que se sometieron a miomectomía laparoscópica y robótica. Se encontró que tanto la miomectomía laparoscópica como la robótica proporcionan reducciones significativas en la gravedad de los síntomas asociados con los fibromas y una mejora significativa en la calidad de vida un año después de la intervención. La tasa de mejoría fue comparable para ambos procedimientos. ${ }^{11}$

Sin embargo, a pesar de la evidencia de que la cirugía mínimamente invasiva es preferible a la laparotomía, la mayoría de las miomectomías todavía se realizan mediante laparotomía. La cirugía robótica se introdujo para eliminar o mejorar algunas de las dificultades asociadas con la cirugía laparoscópica. Se sabe que una miomectomía es una cirugía que requiere de suturar en varios planos, en diferentes sentidos y con anulaciones diversas, en donde se planeó que las características de un robot quirúrgico que ayudara a realizar este trabajo serían de gran valor. Por una vasta bibliografía, en la actualidad se ha demostrado que la miomectomía robótica es eficiente y segura, sus resultados son similares a la cirugía laparoscópica, aunque el procedimiento robótico se asocia con un costo más alto.

La introducción de la cirugía robótica ha ampliado las indicaciones de la miomectomía mínimamente invasiva a casos más complejos, que antes se realizaban mediante laparotomía. A pesar de todo, y como lo señala Lonnerfors, no se han publicado ensayos aleatorios, prospectivos y controlados que comparen diferentes abordajes de una miomectomía para hacer un análisis y de ahí derivar las mejores recomendaciones apegadas a la evidencia. ${ }^{12}$

Existen dos metaanálisis que pueden citarse, el primero realizado por lavazzo y cols. que tenía como premisa demostrar que la miomectomía asistida por robot era una opción de tratamiento igual de segura y eficaz que la laparoscopía y que la cirugía abierta para la miomatosis uterina. Se encontró que, con respecto a la comparación entre la técnica robótica y laparoscópica, no se encontraron diferencias significativas entre ambos tipos de cirugía.

Se concluye que el abordaje mínimamente invasivo tiene la ventaja de una menor pérdida de sangre, menor necesidad de transfusión y menor estancia hospitalaria. Los resultados a largo plazo requieren aclararse, incluyendo el control del dolor, la fertilidad y las tasas de embarazo posoperatorias, así como las posibles tasas de recurrencia. ${ }^{13}$

Dos años más tarde, Wang y cols. realizaron un nuevo metaanálisis sobre este mismo tema y concluyeron que, en comparación con los abordajes laparoscópicos y abdominal, la cirugía robótica se asocia significativamente con Menores índices de complicaciones, menor tasa de conversiones del procedimiento y menor sangrado operatorio. ${ }^{14}$

El resultado, en términos funcionales después de realizar una miomectomía por cualquier abordaje, es lograr el nacimiento de un bebé sano. En México, el primer reporte de éxito sobre una miomectomía asistida por robot lo realizó el equipo quirúrgico de los autores de este trabajo. ${ }^{14} \mathrm{Sin}$ embargo, en este rubro tampoco existen conclusiones tan drásticas sobre la vía de abordaje en favor de una u otra técnica de acuerdo con la superioridad por sus resultados reproductivos, debido a la ausencia de estudios aleatorizados que comparen los diferentes abordajes operatorios en este sentido.

Por otra parte, en un análisis comparativo del papel de la laparoscopía apoyada con robot en el campo de la cirugía reproductiva, Jayakumaran lista las ventajas y limitaciones reportadas del uso de la robótica en cirugías reproductivas como miomectomía, entre otras. Encontró que la asistencia robotizada en la cirugía reproductiva presentó una disminución de la pérdida de sangre, menos dolor posoperatorio, una estancia hospitalaria más corta y una convalecencia más rápida, mientras que los resultados 
Tabla 2: Resultados transoperatorios de las tres técnicas quirúrgicas.

\begin{tabular}{lccc} 
& $\begin{array}{c}\text { Miomectomía abdominal } \\
(\mathrm{n}=50)\end{array}$ & $\begin{array}{c}\text { Miomectomía laparoscópica } \\
(\mathrm{n}=50)\end{array}$ & $\begin{array}{c}\text { Miomectomía robótica } \\
(\mathrm{n}=50)\end{array}$ \\
\hline Tiempo operatorio $(\mathrm{min})$ & $85(20-188)$ & $90(29-222)$ & $122(42-289)$ \\
Tiempo de consola $(\mathrm{min})$ & $\mathrm{NA}$ & $\mathrm{NA}$ & $109(25-260)$ \\
Tiempo anestésico $(\mathrm{min})$ & $94(35-208)$ & $101(41-235)$ & $118(35-301)$ \\
Sangrado transoperatorio $(\mathrm{mL})$ & $235(20-2,000)$ & $90(50-2,000)$ & $50(50-1,000)$ \\
Estancia intrahospitalaria (días) & $2(2-5)$ & $1(0-4)$ & $1(0-3)$ \\
Transfusión (\%) & $3(6)$ & $1(2)$ & 0 \\
Complicaciones $(\%)$ & 0 & 0 & 0 \\
Conversión a laparotomía $(\%)$ & NA & $2(4)$ & 0 \\
Conversión a laparoscopía (\%) & NA & NA & 0 \\
\hline
\end{tabular}

reproductivos fueron similares en los demás abordajes. De igual forma, encontró que la cirugía robótica fue tan segura y eficaz como la laparoscopía convencional y representa una alternativa totalmente razonable al abordaje abdominal.

Se sugiere que los procedimientos que son técnicamente desafiantes con la laparoscopía convencional se pudieran realizar con asistencia robótica debido a sus ventajas de una mejor visualización y movimientos de EndoWrist ${ }^{\mathrm{TM}}$ (semejantes a la muñeca de la mano humana) que permiten una sutura precisa. Esto ayuda a superar las limitaciones de la laparoscopía, en especial en procedimientos complicados, y puede acortar la curva de aprendizaje de la cirugía mínimamente invasiva. Se justifican así los estudios controlados y aleatorios que comparen los resultados a corto y largo plazo para fortalecer el papel de la intervención robótica en el campo de la cirugía reproductiva. ${ }^{15}$

En cuanto a la eficiencia con buenos resultados, características fundamentales en los procedimientos quirúrgicos, existe la pregunta de que ihasta qué número de miomas sería posible retirar mediante cirugía mínimamente invasiva?, en particular en cirugía robótica. Kim y colaboradores ${ }^{16}$ demostraron que es factible realizar una miomectomía robótica en pacientes con hasta 20 miomas, conservando la eficiencia y los buenos resultados posoperatorios, y representa incluso un procedimiento más rápido que la miomectomía abierta con la que se comparó en el estudio. ${ }^{17}$

Un punto muy incisivo que se debe señalar es que, en México, siendo hasta el momento una economía emergente, es fundamental mantener un costo controlado en los procesos operatorios sin escatimar los buenos resultados. La gran mayoría de los estudios antes mencionados y disponibles en la actualidad, han hecho las miomectomías laparoscópicas con asistencia robótica con cuatro brazos, es decir un puerto central para la cámara y tres puertos robóticos, más los accesorios necesarios que por lo general son al menos uno más. Eso confiere ciertas ventajas operatorias, pero la mayoría de los casos pocas ventajas cosméticas, y si se trata de invadir lo menos posible, sería prudente utilizar los menos puertos posibles. En este trabajo, las miomectomías robóticas todas fueron realizadas con tres puertos robóticos en total; un puerto central para la cámara y dos brazos robóticos solamente. Se puede variar entre uno o ningún puerto accesorio extra. Eso desafía aún más cualquier plan quirúrgico, pero brinda mayor estética, menor invasión con una contención de costos en beneficio de todos, paciente, hospital y terceros pagadores si fuera el caso, que es al final lo que se busca, buenos resultados con mínimo acceso.

\section{CONCLUSIONES}

La miomectomía laparoscópica convencional y la asistida por robot demostraron resultados muy similares en cuanto estancia intrahospitalaria e índice de complicaciones. Sin embargo, en cuanto a pérdida sanguínea la cirugía robótica mostró menos sangrado transoperatorio. Es necesario realizar estudios prospectivos para determinar las ventajas específicas entre una y otra técnicas. La miomectomía laparoscópica apoyada mediante robot con sólo tres brazos, en lugar de los cuatro utilizados habitualmente, es una técnica segura y efectiva para el tratamiento de miomatosis uterina en pacientes con deseos de fertilidad futura.

\section{REFERENCIAS}

1. Khaund A, Lumsden MA. Impact of fibroids on reproductive function. Best Pract Res Clin Obstet Gynaecol. 2008; 22: 749-760.

2. Gobern JM, Rosemeyer CJ, Barter JF, Steren AJ. Comparison of robotic, laparoscopic, and abdominal myomectomy in a community hospital. JSLS. 2013; 17: 116-120. doi: 10.429 3/108680812X13517013317473.

3. Cook H, Ezzati M, Segars JH et al. The impact of uterine leiomyomas on reproductive outcomes. Minerva Ginecol. $2010 ; 62: 225-236$. 
4. Sunkara SK, Khairy M, El-Toukhy T et al. The effect of intramural fibroids without uterine cavity involvement on the outcome of IVF treatment: a systematic review and meta-analysis. Hum Reprod. 2010; 25: 418-429.

5. Rikhraj K, Tan J, Taskin O, Albert AY, Yong P, Bedaiwy MA. The impact of noncavity-distorting intramural fibroids on live birth rate in in vitro fertilization cycles: a systematic review and meta-analysis. J Womens Health (Larchmt). 2020; 29: 210-219. doi: 10.1089/jwh.2019.7813.

6. Pritts EA, ParkerWH, Olive DL. Fibroids and infertility: and updated systematic review of the evidence. Fertil Steril. 2009; 91: 1215-1223.

7. Galliano D, Bellver J, Días-García C, simón C, pedllicer A. ART and uterine pathology: how relevant is the maternal side for implantation? Hum Repd Update. 2015; 21: 13-38.

8. Gingold JA, Gueye NA, Falcone T. Minimally invasive approaches to myoma management. J Minim Invasive Gynecol. 2018; 25: 237-250. doi: 10.1016/j. jmig.2017.07.007.

9. Laughlin-Tommaso SK, Lu D, Thomas L et al. Short-term quality of life after myomectomy for uterine fibroids from the COMPARE-UF Fibroid Registry. Am J Obstet Gynecol. 2020; 222: 345.e1-345.e22. doi: 10.1016/j. ajog.2019.09.052.

10. Wu CZ, Klebanoff JS, Tyan P, Moawad GN. Review of strategies and factors to maximize cost-effectiveness of robotic hysterectomies and myomectomies in benign gynecological disease. J Robot Surg. 2019; 13: 635-642. doi: 10.1007/s $1701-019-00948-9$.
11. Takmaz O, Ozbasli E, Gundogan S et al. Symptoms and health quality after laparoscopic and robotic myomectomy. JSLS. 2018; 22: e2018.00030. doi: 10.4293/ JSLS.2018.00030.

12. Lonnerfors C. Robot-assisted myomectomy. Best Pract Res Clin Obstet Gynaecol. 2018; 46: 113-119. doi: 10.1016/j. bpobgyn.2017.09.005.

13. lavazzo C, Mamais I, Gkegkes ID. Robotic assisted vs laparoscopic and/or open myomectomy: systematic review and meta-analysis of the clinical evidence. Arch Gynecol Obstet. 2016; 294: 5-17. doi: 10.1007/s00404016-4061-6.

14. Wang T, Tang H, Xie Z, Deng S. Robotic-assisted vs. laparoscopic and abdominal myomectomy for treatment of uterine fibroids: a meta-analysis. Minim Invasive Ther Allied Technol. 2018; 27: 249-264. doi: 10.1080/13645706.2018.1442349.

15. Rivas-López R, Durón-Padilla R, Romero-Hernández S, Audifred-Salomón J, Hernández-Denis JA. Miomectomía laparoscópica asistida por robot y embarazo. Reporte de caso. Ginecol Obstet Mex. 2016; 84: 194-200.

16. Kim H, Shim S, Hwang Y, Kim M, Hwang H, Chung Y et al. Is robot-assisted laparoscopic myomectomy limited in multiple myomas? a feasibility for ten or more myomas. Obstet Gynecol Sci. 2018; 61: 135-141.

17. Jayakumaran J, Patel SD, Gangrade BK, Narasimhulu DM, Pandian SR, Silva C. Robotic-assisted laparoscopy in reproductive surgery: a contemporary review. J Robot Surg. 2017; 11: 97-109. doi: 10.1007/s 1701-017-0682-4. 